ISSN 1991- 8690

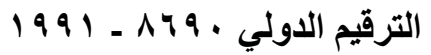

Website: http://jsci.utq.edu.iq

Email: utjsci@utq.edu.iq

تسجيل جديد للمثقوية (1946) Haematotrephus nittanyense (Zeliff متطفلة على الجهاز التفسي

لطيور الكربوع Himantopus himantopus وطيور الزقزلق ابيض الذنب Chettusia leucura في العراق -

نثبلة رشيد الكسار \author{
ذي قار \\ صبيح هليل المياح \\ قسم علوم الحياة ـ كلية التربية للعلوم الصرفة ــ ـ جامعة البصرة \\ E-mail: basim_abdulah@yahoo.com
}

باسم هاثم عبد الله

الخلاصة:

عزلت اليققوبة Haematotrephus nittanyense من الجهاز التتفسي لطيور الكرسوع Himantopus himantopus و طيور الزفزاق ابيض

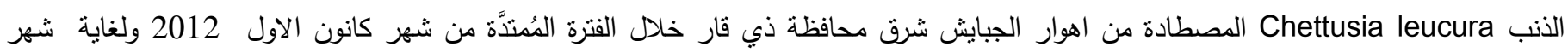

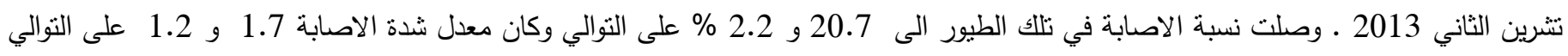

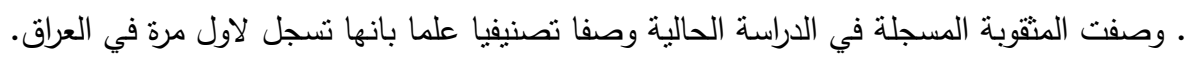

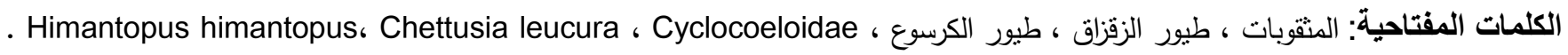

\title{
New record of Haematotrephus nittanyense (Zeliff ,1946) Parasitized in the respiratory system of Black-winged Stilt Himantopus himantopus and White tailed Plover Chettusia leucura birds in Thi Qar Province - Iraq \\ Sabeeh H. Al-Mayah Basim H. Abdullah Nothiala R. Al- kassar \\ Biology Dept .. Education coll . for p. Sci.. Basra Univ. \\ E-mail: basimR-Rabdulah@ yahoo.com
}

Abstract:

Digenetic trematode, Haematotrephus nittanyense is isolated from respiratory system of Black-winged Stilt Himantopus himantopus and White-tailed Plover Chettusia Leucurus,shot in Al- Chibayish marshes , Eastern of Thi Qar province from December 2012 to November 2013 .The Prevalence in those birds are 17.03, $2.15 \%$ respectively . Mean intensity of infection $2.8,1.2$ respectively.. This trematode is recorded for the first time in Iraqi birds,taxonomically described and illustrated. 
AFA الكحول - فورمالين - حامض الخليك) و حفظت في (الكحل

المقدمة:

محلول الكحول والكلسرين (Garcia and Ash , 1979) • استخرجت فيما بعد ووضعت على شرائح زجاجية ، وضحت

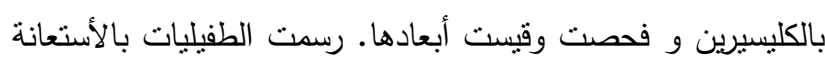

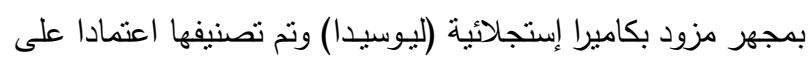

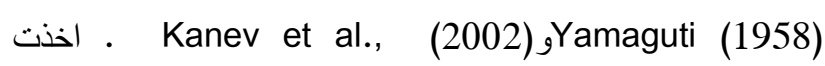
القياسات بالمليمتر ووضعت المعدلات بين قوسين.

النتائج::

Haematotrephus وجد 48 نموذجا من المنقوبة

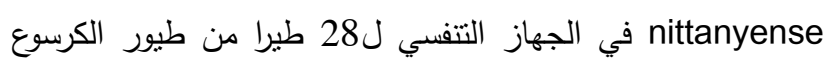

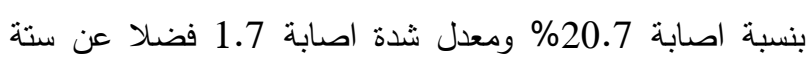

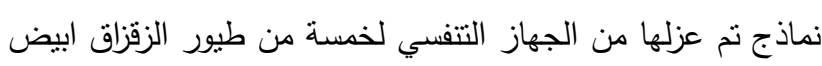

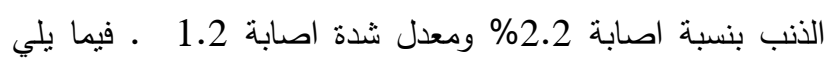

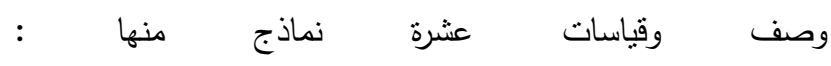

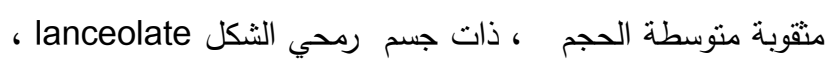

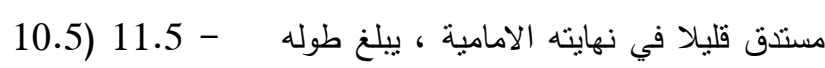

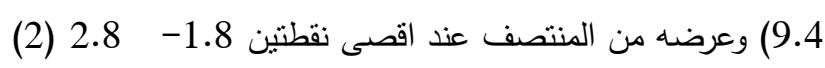

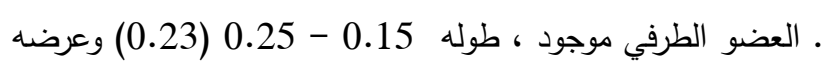
0.2 - 0.3

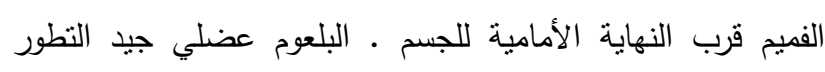

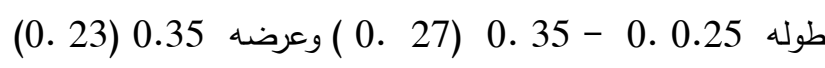
0.20 ويتحدان قرب النهاية الخلفية للجسم لتصبح الأمعاء حلقية الثنكل .

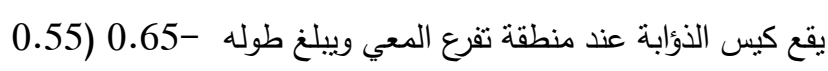

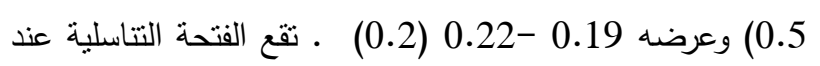
النهاية الخلفية للبلعوم في الناحية البطنية ـ الخصيتان غير منتظمتا

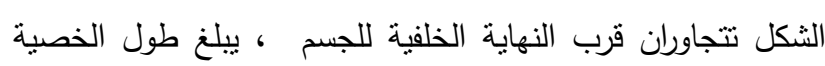

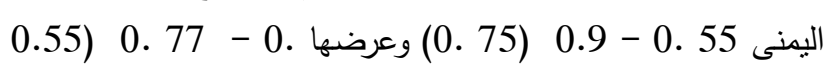

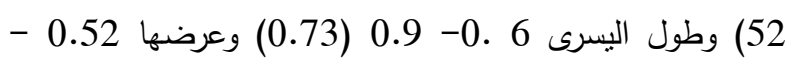

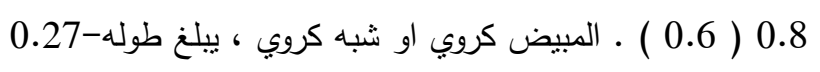

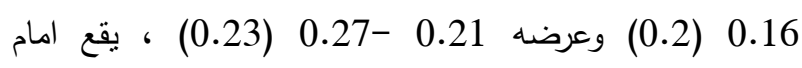
الخصيتين بقليل مشكلا مثلثا معهما. تمند الغدد المحية من امام

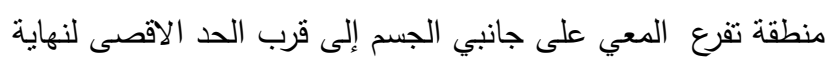

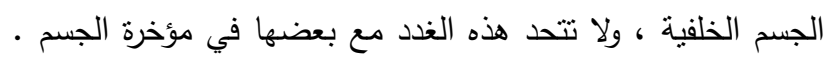

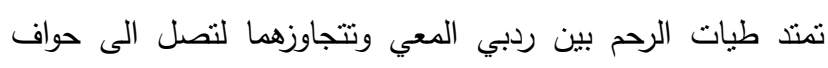

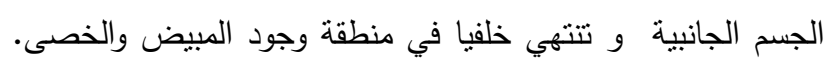
البيوض هلالية الثكل تقريبا ،لها تقعر بسيط في احد جوانبها ،

تضم عائلة Cyclocoeloidae مثقوبات ثنائية المضيف تتطفل بصورة رئيسية في الجهاز التتفسي للطيور ولاسيما المائية

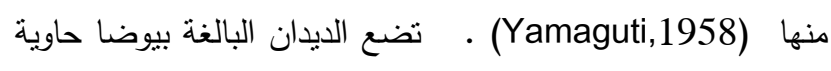
على مهدبات تفقس بعد طرحها في الماء في غضون فترة ولاء وجيزة

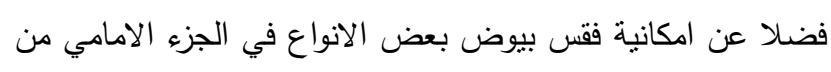

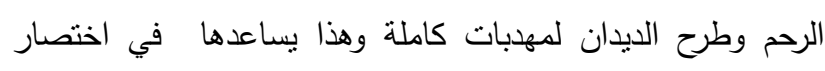

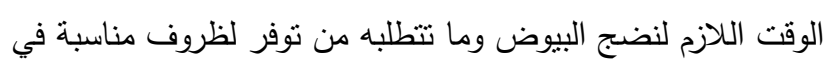

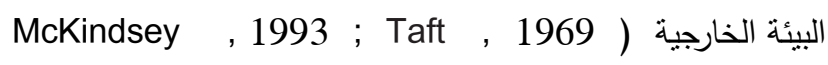

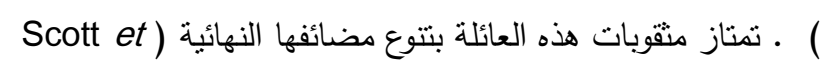

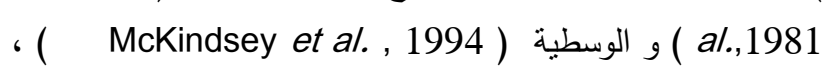
فضلا عن كونها عالمية الانتشار ومسجلة في مناطق منتوعة

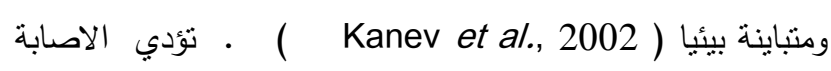
بمنقوبات عائلة Cyclocoeloidae الى ظهور بعض الاعراض

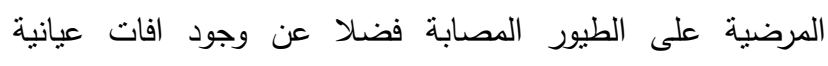
وتغييرات نسجية في بعض الانسجة والاعضاء الحيوية لتلك الطيور ( ) (Scott , 1980 ; McLaughlin , 1977 )

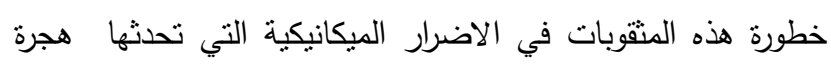

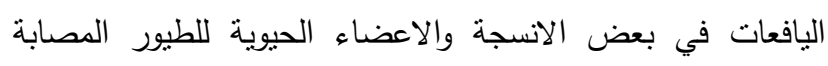

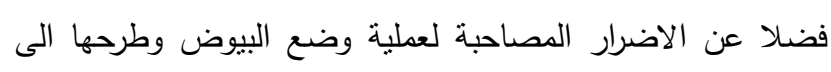

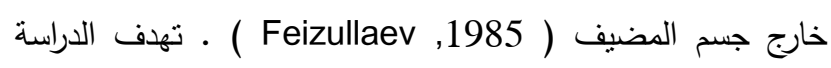
الحالية الى تسليط المزيد من الضوء على مثقوبات هذه العائلة

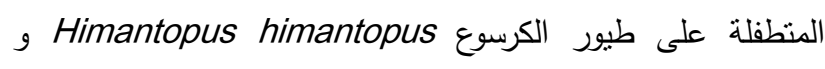

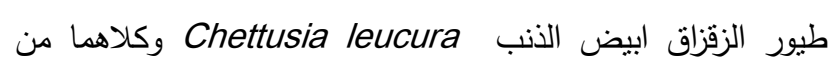

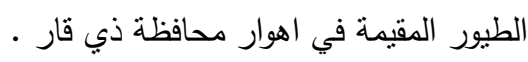

\section{المواد وطرائق العمل:}

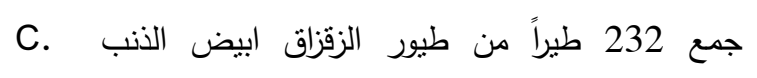

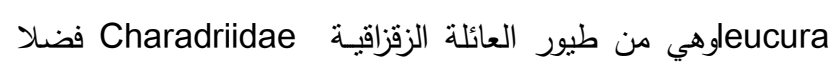

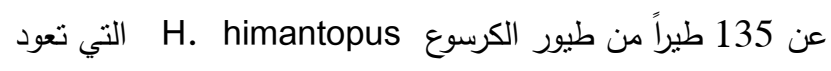

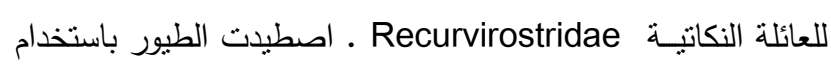

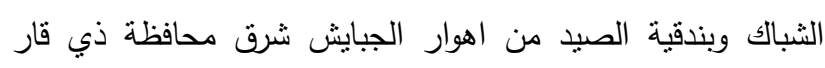

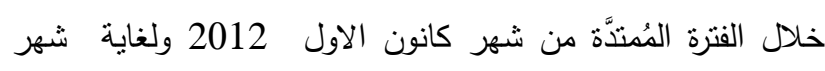
تشرين الثاني 2013 ـ نقلت الطيور الى المختبر اذ صُنفت اعتماداً

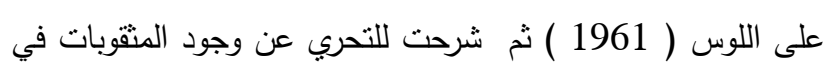

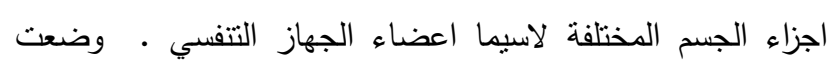
الديدان الحية بعد عزلها بين شريحتين زجاجيتين في المحلول

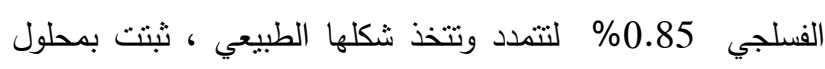


(u) ، كيس الذؤابة (c.)cecum (c.s.) sac

(o.) ، الرحم uterus

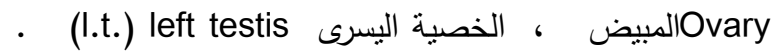

(r.t.) right testis الخصية اليمنى

\section{المناقشة:}

يعد ظهور هذا النوع في الدراسة الحالية أول تسجيل للنوع في

العراق · تتطابق صفات هذه المنقوبة الى حد كبير مع صفات

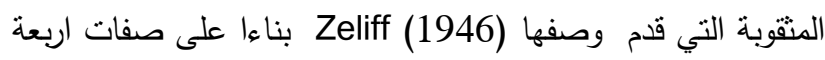

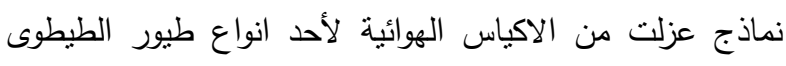
Tringa solitaria solitaria

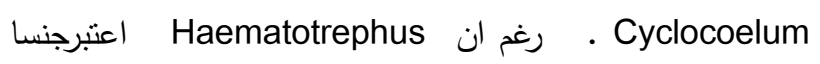
ثانويا تابعا لجنس

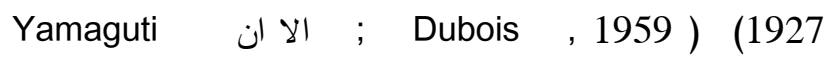
(1971) اشار الى Haematotrephus كنس مستقل ووضع

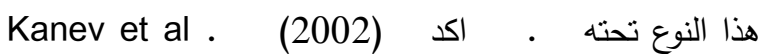
استقلال جنس Haematotrephus الذي يتميز افراده بوجود

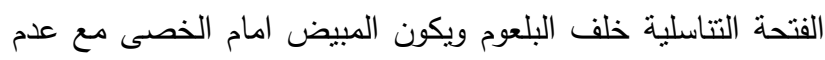

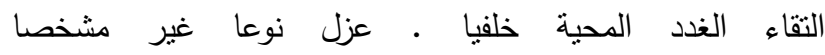
Haematotrephus بعود الى جنس Haematotrephus sp. في العراق من طيور الزقزاق ابيض الذنب C. leucura والكرسوع

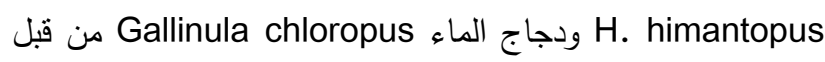
التميمي ( 2013 ) وتميز بكون الخصى فيه مترادفة بيضوية او اوناء كروية الثكل تختلف عن الخصى المتجاورة وغير المنتظمة الثكل التي تعود للنوع الحالي فضلا عن اختلاف النوعين في معظم القياسات التي تمثل ابعاد الجسم وأعضاءه الداخلية وكبر حجم

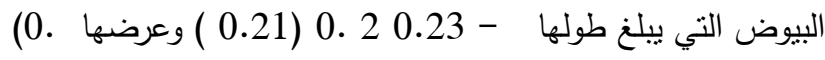

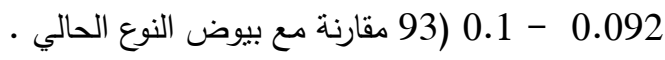

طولها 0.11 - 0.15 (0.13 ) وعرضها 0.065 - 0.09 (0.075) ويفقس بعضها في الجزء الامامي من الرحم . (0.13 ) وعرطن

الموقع التصنيفي

Kanev et al., (2002) و اعتمادا على (1958) Yamaguti

Kingdom : Animalia

Phylum : Platyhelminthes

Class : Trematoda

Subclass : Digenea

Order : Strigeida

Superfamily : Cyclocoeloidea

Family: Cyclocoeloidae

Genus : Haematotrephus

Species: Haematotrephus nittanyense (Zeliff , 1946).

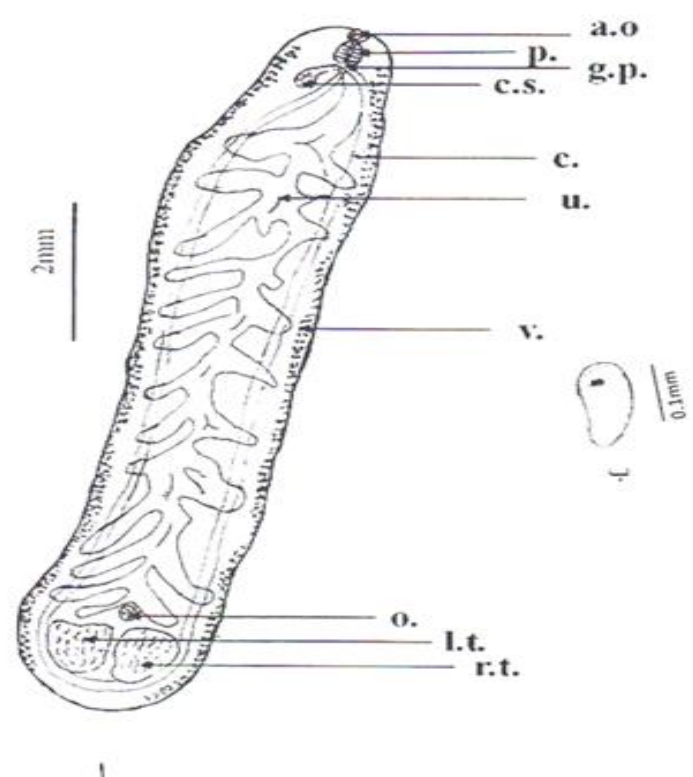

Haematotrephus nittanyense المثقوبة

$$
\text { أ- البالغة }
$$

(p.) pharynx ، العضو الطرفي ب.o.) anterior organ

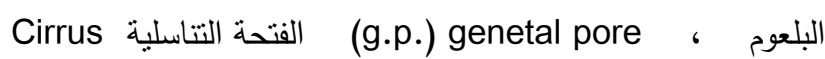


Taft.S.J. (1969). Host-parasite relationships of five species of cyclocoelids ( Trematoda: Cyclocoelidae). Ph.D. Thesis, Iowa State Univ.

Yamaguti, S. (1958). Systema Helminthum, Vol. I. The trematodes of

vertebrates, Parts I Intersci. Publ. Inc. Ltd., New York.

Yamaguti, S. (1971). Synopsis of Digenetic Trematodes of Vertebrates, Volume I, Keigaku Publishing Company, Tokyo, Japan.

McLaughlin, J. D. (1977). The migratory route of Cyclocoelum mutabile Trematoda: Cyclocoelidae). In the American coot, Fulica americana . Can. J. Zool., 55.

McKindsey, C.W. (1993). Lab and field studies on the establishment of

Zeliff, C.C. (1946). A new species of Cyclocoelum, a trematode from the eastern solitary sandpiper. The Ohio J. of Sciences, 46 .
References

المصادر

التميمي،التفات عامر (2013) ـ الديدان المنطفلة في بعض الطيور

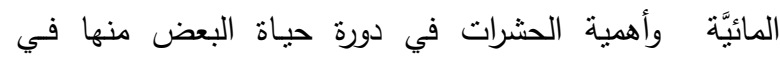

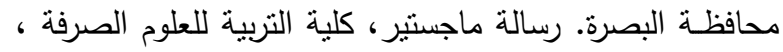

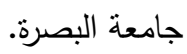

اللوس، بشير (1961) ـ الطيور في العراق. مطبعة الرابطة ، بغداد.

الجزء الثانسي.

Dubois, G. (1959). Revision des Cyclocoelidae Kossack, 1911. Revue Suisse de Zoologie 66.

Feizullaev, N. A. (1985). Pathogenic effect of the trematode Cyclocoeloidea on its hosts. Parazitologiia, 19(3).

Garcia, L.S. and Ash,L.R. (1979) . Diagnostic Parasitology:Clinical Laboratory Manul. $2^{\text {nd }}$ ed .,C.V.Mosby Co.,St.Louis.

Joyeux, C.E. and Baer, J. (1927). Notes sur les Cyclocoelidae. Bulletin de la Société Zoologique de France 52.

Kanev, I., Radev, V. and Fried, B. (2002). Family Cyclocoelidae Stossich, 1902. In: Gibson, D. I, Jones, A. \& Bray, R. A. (Ed.), Keys to the Trematoda. Volume 1. U. K., 131 CABI Publishing and The Natural History Museum, London.

Cyclocoelum mutabile (Zeder, 1800) (Digenea) infections in snail communities. M.Sc. thesis, Concordia Univ., Montreal.

[5]- McKindsey , C. W. ; Goring , J. K. and McLaughlin, J.D. (1994). In vivo and in vitro studies on the viability and the infectivity to coots, Fulica americana of Cyclocoelum mutabile metacerariae from three species of snails. Can. J. of Zool., 72.

Scott,M.E.(1980). A comparative field and laboratory investigation of Typhlocoelum cucumerinum Digenea Cyclocoelidae in various duck and snail hosts. Ph.D. Thesis , Institute of parasitology , McGill Univ.,Montreal.

Scott,M .; Mclaughlin, J. and Rau, M. (1981). Experimental studies on the taxonomic relationships in the subfamily Typhlocoelinae (Digenea: Cyclocoelidae).System. Parasito., 3. 